\title{
The California environmental DNA "CALeDNA" program
}

2 Rachel S Meyer1*, Emily E Curd1, Teia Schweizer1, Zack Gold1, Dannise Ruiz Ramos2,

3 Sabrina Shirazi3, Gaurav Kandlikar1, Wai-Yin Kwan1, Meixi Lin1, Amanda Freise1, Jordan

4 Moberg-Parker1, Miroslava Munguia Ramos4, Beth Shapiro3, Jason P Sexton2, Lenore Pipes5,

5 Ana Garcia Vedrenne1, Maura Palacios Mejia1, Emma L Aronson6, Tiara Moore1, Rasmus

6 Nielsen5, Harris Lewin4, Paul Barber1, Jeff Wall6, Nathan Kraft1, Robert K Wayne1*

8 1. University of California - Los Angeles

9 2. University of California - Merced

10 3. University of California - Santa Cruz

11 4. University of California - Davis

12 5. University of California - Berkeley

13 6. University of California - Riverside

14 7. University of California - San Francisco

Corresponding authors: Robert Wayne (rwayne@g.ucla.edu) and Rachel S Meyer

Glossary at the end

23 Global change is leading to habitat shifts that threaten species persistence throughout

24 California's unique ecosystems. Baseline biodiversity data provide opportunities for 25 ecosystems to be managed for community complexity and connectivity. In 2017, the

26 University of California Conservation Genomics Consortium launched the California

27 Environmental DNA (CALeDNA) program, a community science initiative monitoring

28 California's biodiversity through environmental DNA (eDNA)—DNA shed from

29 organisms through fur, mucus, spores, pollen, etc. Community scientists collect soil and

30 sediment samples, then researchers analyze the eDNA in the samples and share results

31 with the public. The results are catalogues of thousands of organisms per sample, 
33 inventories in a platform designed for the public and researchers alike, as well as user-

34 friendly analysis tools and educational modules. Here, we present CALeDNA as a

35 scalable community science framework that can harmonize with future biodiversity

36 research and education initiatives.

\section{Introduction}

The Earth is facing unprecedented threats to its ecosystems due to climate

42 change, habitat destruction, pollution and other anthropogenic factors. With the $6^{\text {th }}$ mass

43 extinction of life upon us (see Ceballos and Ehrlich, 2018), policymakers and the public

44 need more information to address the grand challenges of how to protect, conserve and

45 restore the health of vital ecosystems that provide food, medicines, raw materials,

46 energy, and cultural attributes essential to human survival and well-being.

In California, one of three North American biodiversity hotspots (Myers et al.,

48 2000; www.cepf.net), 40 million people must find a way to thrive while protecting

49 biodiversity. The economy of California, now ranked fifth in the world, relies heavily on

50 natural resources industries; the state ranks first in recreation tourism, second in

51 seafood production, third in lumber production, and has 39 mined minerals that only

52 occur in commercial quantities in our state (to learn more see

53 www.conservation.ca.gov).

Inventories of California's biodiversity are used to maintain these myriad

55 ecosystem services residents rely on. However, detailed biodiversity data is hard to

56 track across space and time. Fortunately, the past decade has witnessed an impressive 
57 rise in grassroots 'community science' (syn citizen science) campaigns to gather

58 biodiversity data, such as through 'bioblitzes' that monitor species presence or seasonal

59 changes in organismal behavior, interactions, or development. While most community

60 science initiatives are focused on gathering data, we argue that the state of California is

61 a 'living laboratory' to testbed a feedback loop between the public and researchers,

62 where all are engaged in data analysis and interpretation. With numerous world-class

63 research institutions as well as curated living and ex situ natural history collections, and

$6418 \%$ of the U.S. colleges; hundreds of thousands of people in California already engage

65 with environmental sciences and research (www.bls.gov). In addition, California has a

66 strong naturalist certification program, created by the UC division of Agriculture and

67 Natural Resources, where participation in community science is part of the curriculum.

The University of California (UC) Conservation Genomics Consortium (hereafter

69 "the Consortium") launched in 2016 with support from a UC President's Research

70 Catalyst Award. One aim, connecting research activities across campuses, was to

71 develop a high throughput approach for community science-driven habitat monitoring

72 and characterization using an environmental DNA method. In early 2017, the

73 Consortium launched the statewide community science program called CALeDNA (Cal

74 ' $\left.e e^{\prime} D-N-A\right)$. CALeDNA is a platform for public and multi-institutional engagement in

75 biodiversity data collection and analysis using DNA-based technologies executed in a

76 series of steps (Figure 1). CALeDNA recruits and trains community scientists through

77 its website, then coordinates soil and sediment collection using sampling kits and a

78 phone app. Natural areas such as in the UC Natural Reserve System are sampled,

79 analyzed for eDNA, and results are posted online in an interactive website and shared

80 with natural areas managers and stakeholders. 
Diverse communities of researchers and the public have helped develop the

82

83

84

85 research questions and the functionality of CALeDNA. Several California institutions with their own community science programs have partnered to organize bioblitzes and plan research projects (see section 4). Now, the program is focused on building an inclusive network with land managers, policy informers, naturalists, students, and university research scientists, as people are coming together to participate in the analysis of the open results and use the information to address grand challenges of how to steward ecosystems.

\section{2. eDNA: the new biodiversity monitoring tool?}

$$
\text { Environmental DNA is a promising solution to the challenge of monitoring }
$$
marine, terrestrial and freshwater ecosystems (Bohmann et al. 2014; Thomsen and Willerslev 2015). eDNA survey methods rely on all organisms shedding DNA as they live and decay, and these DNA molecules can be isolated, sequenced, and identified (Taberlet et al. 2012). An eDNA-based inventory of a location is a kind of forensic reconstruction of the local organismal community (Thomsen and Willerslev 2015). DNA persists in surface soils and shallow sediments for variable lengths of time (mere days in the ocean, Lafferty et al., 2018; weeks or even several years in terrestrial environments, Barnes and Turner 2016). In all ecosystems, temperature, UV light, microbial metabolic activity, and eDNA shedding rates play complex roles in the production, movement, and degradation rates of eDNA (Barnes and Turner 2016; Deiner et al. 2017). Under certain conditions, like the bottom of a lake, eDNA may be protected from these physical and chemical threats, and may also be sheltered from 
104

105

106

107

108

109

110

111

112

113

114

115

116

consumption by active microorganisms (Palchevskiy and Finkel, 2006), leading to its persistence for up to thousands of years (e.g., Graham et al., 2016).

Next generation (high-throughput) sequencing technologies, such as Illumina

MiSeq, HiSeq or NextSeq systems, substantially reduce the cost of DNA sequence data and allow thousands of different sequences to be retrieved simultaneously. This enabled the emergence of DNA 'metabarcoding', in which specific DNA regions from any organism can be targeted, sequenced, and matched to reference DNA barcodes that communities around the globe have generated from voucher specimens for over three decades. Different barcoding regions are better for different constellations of organisms, but multiple regions can be targeted with metabarcoding, allowing a simultaneous inventory of biodiversity across organismal kingdoms, for costs currently as low as $\$ 35$ a sample, and likely less in the future, as we optimize third generation sequencing technologies, such as PacBio (in progress). For CALeDNA, 4-6 regions are used to obtain metabarcodes from each sample, yielding lists of well over 1000 unique taxa per sample, representing all kingdoms of life. eDNA approaches are ideally suited for intensive and taxonomically broad biodiversity monitoring programs, where they may complement traditional field surveys, such as programs to test the impacts of global and local stressors on California ecosystems (Bohmann et al. 2014; Thomsen and Willerslev 2015).

The promise of eDNA monitoring has led to widespread development and application of this technique including large scale biodiversity monitoring networks (GEOBON and MBON), federal monitoring agencies (USGS and NOAA), local agencies (SCCWRP www.sccwrp.org), and research institutions (NHMLA). California's research communities have pioneered DNA-based environmental assessments (e.g., Southern Sierra Nevada Critical Zone Observatory and the Aronson lab, see Aciego et al., 2017; 
129

130

131

132

133

134

135

136

137

138

139

140

141

142

143

144

145

146

147

148

149

Stanford Center for Ocean Solutions, see Andruszkiewicz et al., 2018). Diverse

researchers and resource managers have been using eDNA approaches to detect and monitor endangered species, track the emergence and spread of invasive species, and inventory biodiversity in a wide variety of habitats from submarine canyons to alpine

forests demonstrating the breadth of applications of this emerging technique. Work thus far has still largely focused on water sampling or focused on limited groups of taxa such as bacteria or fish (as in above two references).

\section{CALeDNA program orientation}

\subsection{Study sites}

Study areas can be chosen in two ways: (1) by researchers with projects, who propose collection in certain areas, habitat types, or transects, and who may organize group eDNA collection events, or (2) by community science volunteer choice.

Volunteers can collect for CALeDNA from anywhere they please as long as they have proper permission such as collection permits or written permission from a landowner.

While obtaining permission to collect eDNA may take time, it has not discouraged volunteers interested in adding an area of their interest to the CALeDNA map (Figure 1). CALeDNA reimburses all permitting fees incurred. This can also benefit groups, for example, one volunteer-a teacher-independently obtained a permit for Vasona Lake Park in Summer 2018, and brought the Youth Science Institute summer camp students to collect. Overall, the contribution of sites by the public and by researchers ensures a diverse sampling, increases awareness of accessible natural areas for all parties, and strives for sufficient sampling to meet research needs that will result in publications. 
At the time of writing this, one third of our samples are from UC Natural

154 Reserves. The UC boasts the largest university reserve system in the world, at 39 (soon

15540 ) reserves totaling over 756,000 acres. Most of these reserves aren't open to the public.

156 UC researchers may visit, accompany volunteers, or even just send volunteers, to hike

157 through and sample eDNA. The reserves are ideal to provide a biodiversity baseline for

158 the state because they include coastal to montane biomes.

159 All reserves have hosted numerous traditional biodiversity surveys, and we use

160 these to assess the extent of overlap between eDNA metabarcoding and traditional

161 sampling, which can illuminate the bias as well as complementarity in eDNA and

162 human surveys. The reserves offer additional abiotic data that may strengthen statistical

163 analyses and models to describe eDNA patterns. These include weather station and

164 tower data, such as that implemented by Institute for the Study of Ecological and

165 Evolutionary Climate Impacts (https: / / iseeci.ucnrs.org), and NASA pre-HyspIRI

166 flights, where for 7 years, data have been collected from pathways intentionally situated

167 over UC reserves.

\subsection{The sampling experience}

Volunteers may join a bioblitz, or may sample a site on their own. In either case,

171 they would receive a sampling kit of gloves, tubes, and an optional meter for collecting

172 abiotic data (Figure 2a). Each sampling kit is used together with an electronic webform

173 for smartphones and tablets or with a paper form. Forms are for the collector to provide

174 important collection metadata (Figure 2b). These metadata fields are more than the

175 minimum information currently required for meeting sample description standards

176 (e.g. NCBI Bioproject), but additional data make samples more likely to be used for 
177

178

179

180

181

182

183

184

185

186

187

188

189

190

191

192

193

194

195

196

197

198

199

200

201

analysis. CALeDNA data standards are inspired by the Global Genome Biodiversity

Network (ggbn.org).

Our webforms are made using the KoBoToolbox (kobotoolbox.org) platform to create and curate webform information. Results are backed up in real time. CALeDNA is dynamic, and different projects may require different metadata. Kobo Toolbox allows multiple forms to be created with the same minimum essential questions.

\subsection{The 'eDNA museum'}

Upon receipt of the collected samples, each eDNA sample tube is treated as a valuable biological research collection. Samples get archived into a $-80^{\circ} \mathrm{C}$ freezer that is part of the permanent "Dickey Collection" at UCLA, or archived in freezers at other UC campuses as satellite collections. We intend for the CALeDNA samples to be used to track environmental change over the next hundred years. When samples are processed and results are published online, the physical locations of the archived samples are reported and archived as part of the sample metadata.

Samples and kit materials are physically returned to UC campuses via pick up, drop off or Fedex. For the latter, we email shipping labels to volunteers so they do not need to pay out of pocket.

We encourage sample return within one week of collection. Many volunteers collect samples over long treks; in these cases, we request they refrigerate samples $\left(4{ }^{\circ} \mathrm{C}\right)$ until they can be shipped back all at once for archiving in our freezers. Tests have shown that freezing and thawing samples causes DNA profiles to vary, but maintaining a stable temperature helps to preserve the balance of DNA profiles (www.earthmicrobiome.org; Thompson et al., 2017). Considering the rapid advancement in technology, and our hopes that these eDNA samples will be used in 
research far in to the future, we chose to avoid adding stabilizing buffers to the samples that may pose unknown effects to the sample integrity. collections increase. Under current funding, we are sequencing $10 \%$ of the samples received and make these results immediately open to the public.

211 sediment from each is pooled into a single tube that is mixed and used for DNA

212 extraction. As a dynamic program, sampling methods may diversify in the future. For 213 example, the Aronson Lab (UCR) is engineering rollers as eDNA surface collectors,

214 along with wearable passive eDNA samplers.

DNA is processed through a series of steps to generate metabarcoding libraries.

216 Because contamination from the sample collector or from the lab is a common problem

217 in eDNA research, sometimes field 'blanks' are collected, and when extracting DNA, a

218 'blank' sample is also extracted as every batch of samples are processed. The details of

219 the DNA preparation pipeline and CALeDNA protocols can be found on our website

220 (www.ucedna.com) in the "researchers" space [DOIs to protocols will be assigned upon

221 acceptance]. Each barcode region we target requires three separate PCR reactions as

222 'technical replicates' that help reduce reaction bias in the results, meaning for 5

223 barcoding regions, there may be 18 reactions per sample. Metabarcode libraries are

224 sequenced on a MiSeq machine that generates paired reads 2 x 300 base pairs long,

225 meaning when put together, each sequence can be up to 600 base pairs. This allows us

226 to use lengthier barcode regions such as a portion of the CO1 marker (Leray et al., 2013) 
227 to inventory animals. We aim to sequence a minimum of 25,000 paired reads for each

228 barcoding region for each sample.

229 DNA data are deposited in the National Center for Biotechnology Information

230 (NCBI) Sequencing Read Archive. These DNA data are processed through a series of

231 software in the Anacapa Toolkit (Curd et al., submitted;

232 https: / / www.biorxiv.org/content/early/2018/12/07/488627) that was specifically

233 developed for CALeDNA's multilocus metabarcoding approach. The toolkit combines

234 state-of-the-art methods and is flexible to handle many kinds of eDNA data. CALeDNA

235 researchers coordinating with eDNA researchers from academic, non-profit (Code for

236 Science and Society), and government spheres to help onboard new user groups to

237 Anacapa, which create opportunities for data integration.

238 Results are a list of taxa and the number of sequences that matched each one in

239 each sample. The taxa may be identified to the level of species or limited to a higher

240 rank such as genus or family, depending on the completeness of DNA barcode

241 reference databases and the number of diagnostic DNA bases for that particular

242 organism. CALeDNA scientists are working to solve this issue in the Nielsen Lab at UC

243 Berkeley, but even in despite of this caveat, plenty of biodiversity patterns can be

244 gleaned from higher taxonomic levels, like family, or from sheer genetic diversity.

3.5. Open data and results

To allow users to track our progress once samples are received, we put the field

248 data collected by the community scientist online shortly after data are received. To

249 make our results open and accessible, the eDNA results are deposited online shortly

250 after processing through Anacapa and removing contaminants. Our impetus for open

251 data is that scientists around the world are increasingly committing to the FAIR data 
252 principles (FORCE11.org) of findability, accessibility, interoperability, and re-usability.

253 However, because endangered species may more easily be poached with help of eDNA

254 leads, the CALeDNA website omits the specific sites where IUCN redlisted species have

255 been found.

256 The Anacapa Toolkit is linked with an interactive results analysis platform called

257 ranacapa (Kandlikar et al., 2018) that allows users to execute the same first-pass

258 biodiversity data analyses of research projects as professional community ecologists

259 typically do, but the automation in ranacapa relieves users of the need to code or use

260 advanced statistical software. Plots and statistics are produced with explanations aimed

261 at the undergraduate level. This enables community science users to reproduce results

262 CALeDNA reports on the website or in scientific journals. Because data and tools are

263 shared early in the analysis stage, community scientists may make some discoveries

264 first, report them to CALeDNA, and through this feedback loop, earn co-authorship on

265 research publications while bringing attention to the biodiversity in areas they care

266 about.

267

\section{CALeDNA research project vignettes}

\subsection{The Pillar Point project: assessing overlap between eDNA and human observation}

271 Our first bioblitz in early 2017 was in collaboration with the California Academy

272 of Sciences (CAS) and the Los Angeles Natural History Museum (NHMLA) to explore a

273 potential complementary trifecta for biodiversity monitoring: human observation

274 (CAS), DNA barcode sequences from local species (NHMLA), and eDNA (CALeDNA).

275 Since 2012, CAS has been running monthly bioblitzes at the Pillar Point Harbor

276 tidepools and adjacent areas within Half Moon Bay 
277 (https://www.inaturalist.org/projects/intertidal-biodiversity-survey-at-pillar-point),

278 which is why this area was chosen. eDNA provides complementary results to human

279 observation (Figure 3; manuscript in preparation;

280 https: / / data.ucedna.com/research_projects / pillar-point).

281 4.2. Point Fermin: do eDNA results improve with local DNA barcoding?

282 NHMLA runs semi-annual bioblitzes in conjunction with Snapshot CalCoast

283 (https: / / www.calacademy.org/calcoast) during low tide at Point Fermin Park in San

284 Pedro, California (Figure 4a). They take photographs and make physical voucher

285 collections as well, which later are DNA barcoded for the CO1 region as part of the

286 DISCO project https:// research.nhm.org/disco/disco.html. eDNA collections

287 concurrent with NHMLA bioblitzes help us assess how much results improve with very

288 local DNA barcoding.

289

\subsection{California macro-ecological patterns}

From April 2017 to July 2017, a series of bioblitzes and independent community

292 science activities in parks and reserves brought in thousands of soil or sediment

293 samples to the CALeDNA collection. CALeDNA scientists selected 278 of these

294 represented latitudinal transects along forest, shrub/scrub, or coastal areas down the

295 state of California. Analysis of sequencing results reveals 25,283 unique taxonomic

296 entries. We are performing different kinds of diversity analyses (e.g. Figure 5) and

297 statistical modeling to ask what environmental factors influence biodiversity.

298

\subsection{Patterns of biodiversity along the California coast}

Together with over two dozen colleagues from California State University

301 campuses and coastal reserves, CALeDNA coordinated two distributed bioblitzes to 
sample along a $1200 \mathrm{~km}$ span of coast from Arcata to San Diego (Figure 4b). Over 80

phyla were identified and now, the team is asking how their presence predicts coastal

304 health and uniqueness. These bioblitzes will be repeated to monitor coastal biodiversity

305 change.

\subsection{Persistence of eDNA in vernal pools}

Vernal pools are temporary wetlands, filled by substantial rainy seasons, special adaptations to pool depth, morphology and geochemistry. CALeDNA researchers from the UC Merced Dawson and Sexton labs are studying eDNA of five vernal pools on the UC Merced Vernal Pool and Grassland Reserve to build a more

313 comprehensive taxon inventory (Figure 4c).

\subsection{Invasive grasses in shrub/open forests} that they invade. The Fort Ord Natural Reserve has supported multi-day bioblitzes that have added nearly 200 samples to the CALeDNA collection with associated metadata of

320 which sites have invasive grasses. UCSC graduate student Sabrina Shirazi is identifying

321 associations between invasive species and the rest of the community detected with eDNA. 
327 to sample sediment from different areas of lagoons (Figure 4e). She is evaluating the

328 ability of eDNA to inventory community species and associate them with

329 environmental stress response. DNA is being used in metabarcoding and also run on a

330 GeoChip (Glomics, Inc) that quantifies the presence of 22,000+ genes involved in stress

331 response and ecosystem functioning.

\subsection{Burn sites}

California has experienced and increase in fires and burn intensity that have devastated areas that are normally spared as refugia. CALeDNA community science volunteers and UC undergraduate classes began sampling paired burned and unburned

337 sites (Figure 4f), and began resampling sites that were affected by fire. This will enable

338 CALeDNA researchers to track biodiversity change after fire.

\section{9. eDNA to describe the desert}

343 detect a biodiversity in desert ecosystems (Figure $4 \mathrm{~g}, \mathrm{~h}$ ). Community scientists like

344 [NAME OBSCURED] and Friends of the Desert Mountains contribute substantial

345 collections for CALeDNA.

\subsection{Exploring eDNA methods}

The Shapiro lab at UCSC has tested how different approaches in preparing metabarcode libraries influence eDNA results that will help us tune methods to make 
have identified amplification enzymes that amplify DNA with less bias (Nichols et al., 2018). They continue to test technical effects on eDNA results.

\section{5. eDNA in undergraduate education}

\subsection{Authentic research in the microbiology classroom}

In Winter 2017, the newly launched CALeDNA initiative began a partnership

with the UCLA Microbiology, Immunology, \& Molecular Genetics (MIMG)

CUREs have been demonstrated to provide a more inclusive avenue for students that might not otherwise have the opportunity to participate in research (Auchincloss et al. 2014). The MIMG CURE is a two-quarter research immersion curriculum in which upper-division undergraduates work in teams to formulate and test their own hypotheses regarding soil microbial ecology using eDNA and traditional bacterial cultivation methods (Shapiro et al. 2015). Using the CALeDNA sample collection kits and eDNA analysis tools, undergraduates have compared the soil microbiomes of

367 California native and invasive plant species, natural and managed ecosystems, and studied the effects of human impact and burning on microbiomes.

Undergraduates connect with graduate students doing related eDNA research who visit the classrooms, and we hope this encourages students to consider graduate

371 careers. This partnership between CALeDNA and MIMG inspired the development of

372 eDNA and microbiology analysis tools spearheaded by graduate students and

373 instructors, such as ranacapa (Kandlikar et al. 2018) and PUMA (Mitchell et al., in

374 review; https://www.biorxiv.org/content/early/2018/11/29/482380). Several MIMG

375 students have joined the CALeDNA labs as research interns. 
Wayne (UCLA) and Shapiro (UCSC). This program aims to educate and encourage undergraduates to enter STEM fields through field-based and flipped learning courses, workshops, and research, where eDNA gives entrée into the diverse natural and social sciences it can inform. An introductory course for freshmen and transfer students debuted in Fall 2018: California's DNA: A Field Course (Figure 6, left). A 4-credit course, Biodiversity in the Age of Humans, is planned for Spring 2019 and will make use of the active learning classrooms at UCLA and UCSC campuses.

394 UCLA and UCSC offered eleven positions for 10-week paid summer research

395 internships to work with 6 different professors after the Institute.

\section{Building a stronger eDNA community}


results and connect with university researchers through our web interface and our bioblitzes. A team of graduate student Information Architects as well an experiences web programmer with a passion for science were crucial to the production of the website. We encourage feedback and ideas for how to serve the community, and how to use eDNA science to inform policy.

In the next phase of the program we will tie CALeDNA into the Earth BioGenome Project (EBP; Lewin et al., 2018). The EBP is a moonshot to sequence the genomes of all eukaryotes on Earth. There are approximately 9000 eukaryotic taxonomic families on Earth (Lewin et al,. 2018), and at least 35,000 species in California. CALeDNA will provide information on where species are distributed and where new 411 species may occur, so that those places may be sampled for the EBP collections. Our research teams are beginning to invent ways to use entire genomes to monitor

413 demographic and evolutionary change with eDNA, not just occurrence.

415 scientists, naturalists, observers, local scientific societies, biological collections and

416 information curators, to help the EBP effort lead to solutions in California. Together,

417 California can build a biodiversity-responsible and DNA-innovative economy to meet

418 the challenges of climate change and a growing population.

420 Acknowledgements: We thank the University of California Office of the President

421 Catalyst Program (CA-16-376437), the Howard Hughes Medical Institute Grant

423 (ZG). We thank Michael Dawson (UC Merced) for contributing to the CALeDNA

424 program design, for coordinating the coastal bioblitzes, and for constructive comments

425 on the manuscript. We thank the following people who have guided the CALeDNA 
426 program: Regina Wetzer, Dean Pentcheff, and Adam Wall of the Natural History

427 Museum Los Angeles, Eric Crandall (CSUMB), Alison Young and Rebecca Johnson of

428 the California Academy of Sciences, Greg Suba (CNPS) and Joseph Miller (UCSC). We

429 thank former interns Amber DeVries, Laura Rabichow, Larysa Bulbenko, Aoife Galvin,

430 Audrey Mahinan, and Eric Beraut. We thank the UCLA UX Team for guiding the web

431 development. We thank all UC Natural Reserve Managers who made CALeDNA

432 collection possible and inspired research questions, and especially acknowledge the

433 contribution of the late Don Canestro (UCSB).

441 Literature Cited:

442

443 Aciego SM, Riebe CS, Hart SC et al. 2017. Dust outpaces bedrock in nutrient supply to

444 montane forest ecosystems. Nature Communications, 8:14800.

445 doi:10.1038/ncomms14800.

447 Andruszkiewicz EA, Starks HA, Chavez FP et al. 2017. Biomonitoring of marine

448 vertebrates in Monterey Bay using eDNA metabarcoding. PLOS ONE, 12:e0176343.

449 doi:10.1371/journal.pone.0176343. 
451 Auchincloss LC, Laursen SL, Branchaw JL et al. 2014. Assessment of course-based

452 undergraduate research experiences: a meeting report. CBE Life Sci Educ, 13:29-40.

453 doi:10.1187/cbe.14-01-0004.

454

455 Barnes MA \& Turner CR. 2016. The ecology of environmental DNA and implications for 456 conservation genetics. Conserv Genet, 17:1-17. doi:10.1007/s10592-015-0775-4.

458 Bohmann K, Evans A, Gilbert MTP et al. 2014. Environmental DNA for wildlife biology 459 and biodiversity monitoring. Trends in Ecology \& Evolution, 29:358-367.

460 doi:10.1016/j.tree.2014.04.003.

462 Ceballos G \& Ehrlich PR. 2018. The misunderstood sixth mass extinction. Science, 463 360:1080-1081. doi:10.1126/science.aau0191.

465 Deiner K, Bik HM, Mächler E et al. 2017. Environmental DNA metabarcoding:

466 Transforming how we survey animal and plant communities. Molecular Ecology, 467 26:5872-5895. doi:10.1111/mec.14350.

469 Gaertner M, Biggs R, Beest MT et al. 2014. Invasive plants as drivers of regime shifts:

470 identifying high-priority invaders that alter feedback relationships. Diversity and

471 Distributions, 20:733-744. doi:10.1111/ddi.12182.

473 GBIF. 2018. “Adding sequence-based identifiers to backbone taxonomy reveals 'dark 474 taxa' fungi". https://www.gbif.org/news/2LrgV5t3ZuGeU2WIymSEuk/adding- 
sequence-based-identifiers-to-backbone-taxonomy-reveals-dark-taxa-fungi. Accessed

$476 \quad$ November 22,2018.

477

478 Graham RW, Belmecheri S, Choy K et al. 2016. Timing and causes of mid-Holocene

479 mammoth extinction on St. Paul Island, Alaska. PNAS, 113:9310-9314.

480 doi:10.1073/pnas.1604903113.

481

482 Hawkes CV, Belnap J, D’Antonio C et al. 2006. Arbuscular Mycorrhizal Assemblages in

483 Native Plant Roots Change in the Presence of Invasive Exotic Grasses. Plant Soil,

484 281:369-380. doi:10.1007/s11104-005-4826-3.

486 Kandlikar GS, Gold ZJ, Cowen MC et al. 2018. ranacapa: An R package and Shiny web

487 app to explore environmental DNA data with exploratory statistics and interactive

488 visualizations. F1000Research, 7:1734. doi:10.12688/f1000research.16680.1.

490 Lafferty KD, Benesh KC, Mahon AR et al. 2018. Detecting Southern California's White

491 Sharks With Environmental DNA. Front Mar Sci, 5. doi:10.3389/fmars.2018.00355.

493 Leray M, Yang JY, Meyer CP et al. 2013. A new versatile primer set targeting a short

494 fragment of the mitochondrial COI region for metabarcoding metazoan diversity:

495 application for characterizing coral reef fish gut contents. Front Zool, 10:34.

496 doi:10.1186/1742-9994-10-34.

497

498 Lewin HA, Robinson GE, Kress WJ et al. 2018. Earth BioGenome Project: Sequencing life

499 for the future of life. PNAS, 115:4325-4333. doi:10.1073/pnas.1720115115. 
501 Myers N, Mittermeier RA, Mittermeier CG et al. 2000. Biodiversity hotspots for

502 conservation priorities. Nature, 403:853-858. doi:10.1038/35002501.

503

504 Nichols RV, Vollmers C, Newsom LA et al. 2018. Minimizing polymerase biases in 505 metabarcoding. Mol Ecol Resour, 18:927-939. doi:10.1111/1755-0998.12895.

507 Palchevskiy V \& Finkel SE. 2006. Escherichia coli Competence Gene Homologs Are 508 Essential for Competitive Fitness and the Use of DNA as a Nutrient. J Bacteriology, 509 188:3902-3910. doi:10.1128/JB.01974-05.

511 Shapiro C, Moberg-Parker J, Toma S et al. 2015. Comparing the Impact of Course-Based 512 and Apprentice-Based Research Experiences in a Life Science Laboratory Curriculum. J

513 Microbiol Biol Educ, 16:186. doi:10.1128/jmbe.v16i2.1045.

515 Taberlet P, Coissac E, Hajibabaei M et al. 2012. Environmental DNA. Molec Ecol, 516 21:1789-1793. doi:10.1111/j.1365-294X.2012.05542.x.

518 Thompson LR, Sanders JG, McDonald D et al. 2017. A communal catalogue reveals

519 Earth's multiscale microbial diversity. Nature, 551:457-463. doi:10.1038/nature24621.

521 Thomsen PF \& Willerslev E. 2015. Environmental DNA - An emerging tool in

522 conservation for monitoring past and present biodiversity. Biol Cons, 183:4-18.

523 doi:10.1016/j.biocon.2014.11.019. 
bioRxiv preprint doi: https://doi.org/101101/503383; this version posted January 4, 2019. The copyright holder for this preprint (which was not certified by peer review) is the author/funder, who has granted bioRxiv a license to display the preprint in perpetuity. It is made available under aCC-BY-NC-ND 4.0 International license.

525 van der Putten WH, Klironomos JN \& Wardle DA. 2007. Microbial ecology of biological

526 invasions. ISME J, 1:28-37. doi:10.1038/ismej.2007.9.

527

528

529

530

531

532

533

534

535

536

537

538

539

540

541

542

543

544

545

546

547

548 
Figure 1. Top: Flowchart of how CALeDNA works. Bottom: Map of California showing

550 the sites sampled by volunteers, and the proportion of samples for which eDNA results

551 are publicly available.. Blue spots indicate the locations of UC Natural Reserves. Results

552 from different organismal groups can be queried on the www.ucedna.com 'explore

553 data' pages and plotted against different maps (example here shown is the proportion

554 of silt in soils). The intention is for the user to do qualitative data exploration and

555 generate hypotheses based on spatial patterns.

557 Figure 1.

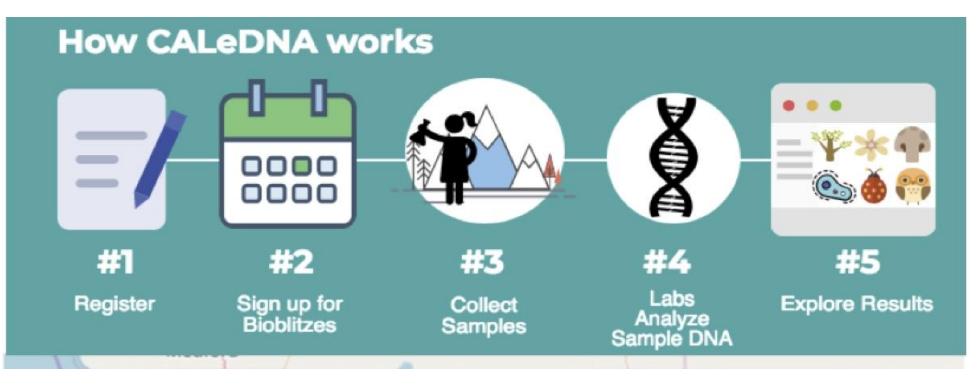

.

(
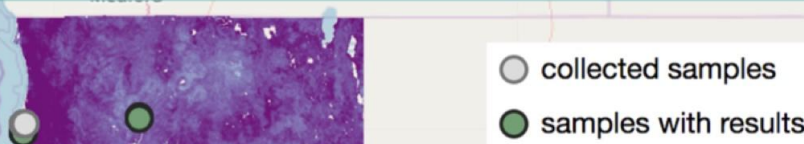

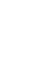


573 Figure 2. a. CALeDNA kit contents, including a pair of gloves, a set of three tubes for

574 biological replicates packed inside a Whirl-Pak bag to protect tubes, a straw to sample

575 sediment or to move large debris to expose topsoil, a ruler, and a meter. b. Webform

576 fields the collector fills in when sampling a site.

578 Figure 2.

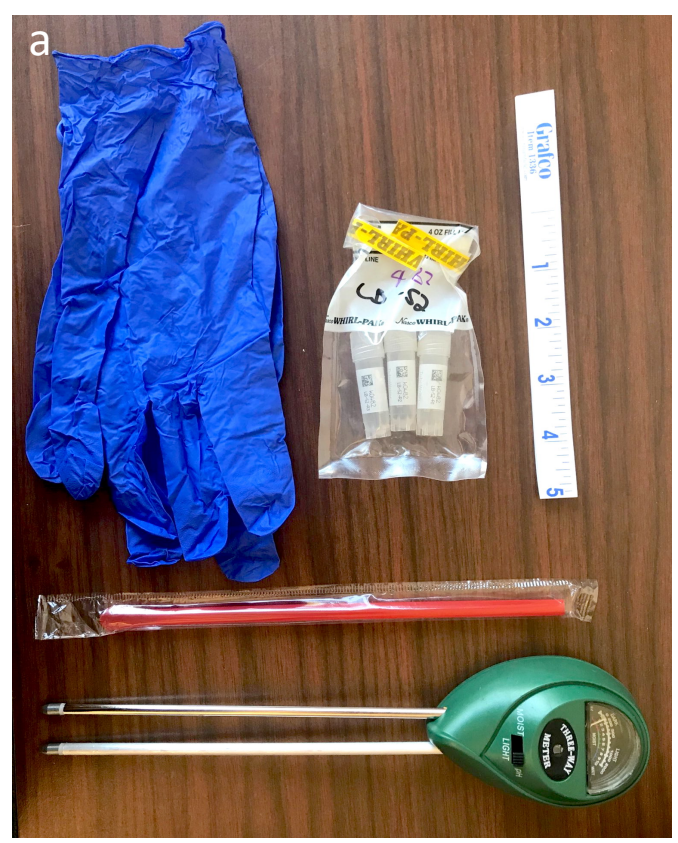

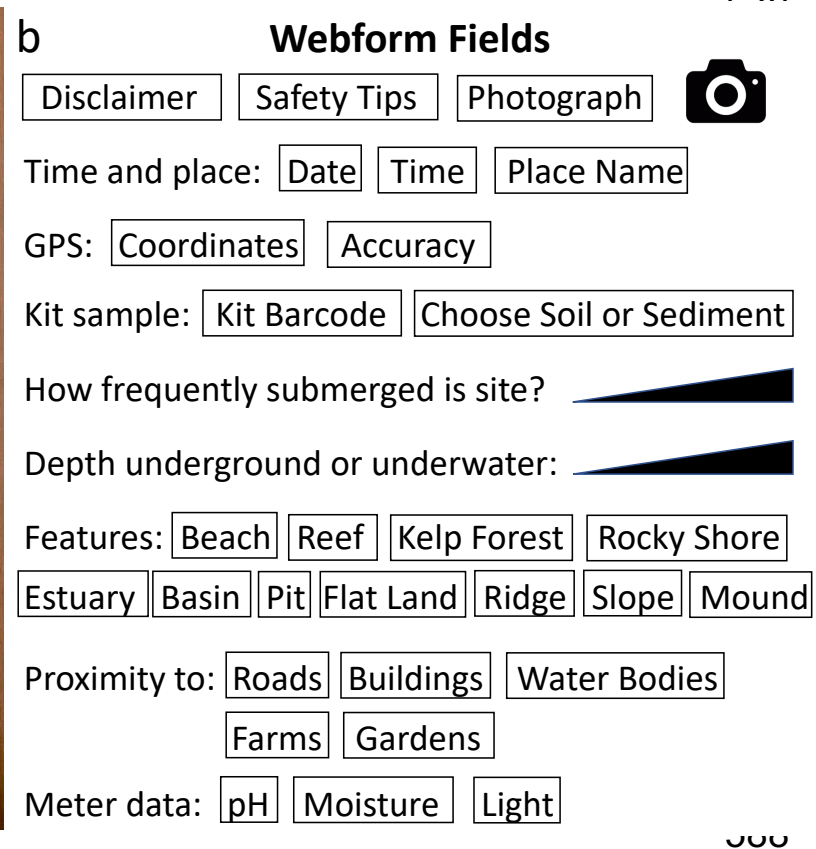


598 Figure 3. Pillar Point project overview. a. The project is an test of how observations,

599 largely facilitated by the California Academy of Sciences iNaturalist program, integrate

600 with local DNA barcoding efforts done by the Natural History Museum of Los Angeles

601 Diversity Initiative for the Southern California Ocean (DISCO), and eDNA results from

602 CALeDNA bioblitzes. These initiatives can cross-inform each other to broaden

603 awareness of biodiversity that can be monitored through community science. $b$.

604 Comparison of GBIF data, containing iNaturalist records and all other non-eBird

605 observations, to eDNA. c. The Pillar Point project divides the region into three sections:

606 an embankment (yellow), an unprotected exposed area containing accessible tidepools

607 (blue), and the State Marine Protected Area (SMCA; red). The pins are eDNA sampling

608 locations. The circles are GBIF observation records, colored by kingdom (blue is animal,

609 green is plant, red is fungus). d. Area diversity showing the number of unique taxa

610 observed from GBIF versus eDNA from the three sections of Pillar Point. Overlap is

611 shared taxa. Colors for the sections are as in C. 
bioRxiv preprint doi: https://doi.org/10.1101/503383; this version posted January 4, 2019. The copyright holder for this preprint (which was not certified by peer review) is the author/funder, who has granted bioRxiv a license to display the preprint in perpetuity. It is made available under aCC-BY-NC-ND 4.0 International license.

\section{Figure 3.}

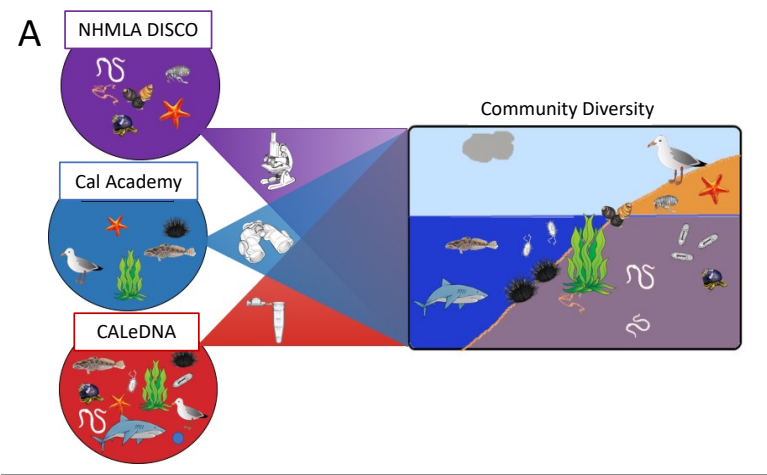

B Taxa reported at Pillar Point by eDNA and GBIF

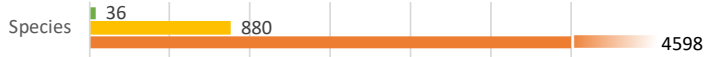

Genera $\quad$\begin{tabular}{r|r}
\hline & -610
\end{tabular}

Families 117358

1371

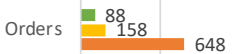

Classes 140

\begin{tabular}{l|l} 
Phyla & 19 \\
192 & 82
\end{tabular}

$\begin{array}{lllll}0 & 500 & 1000 & 1500 & 2000\end{array}$

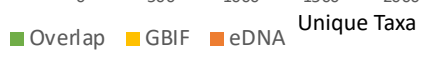
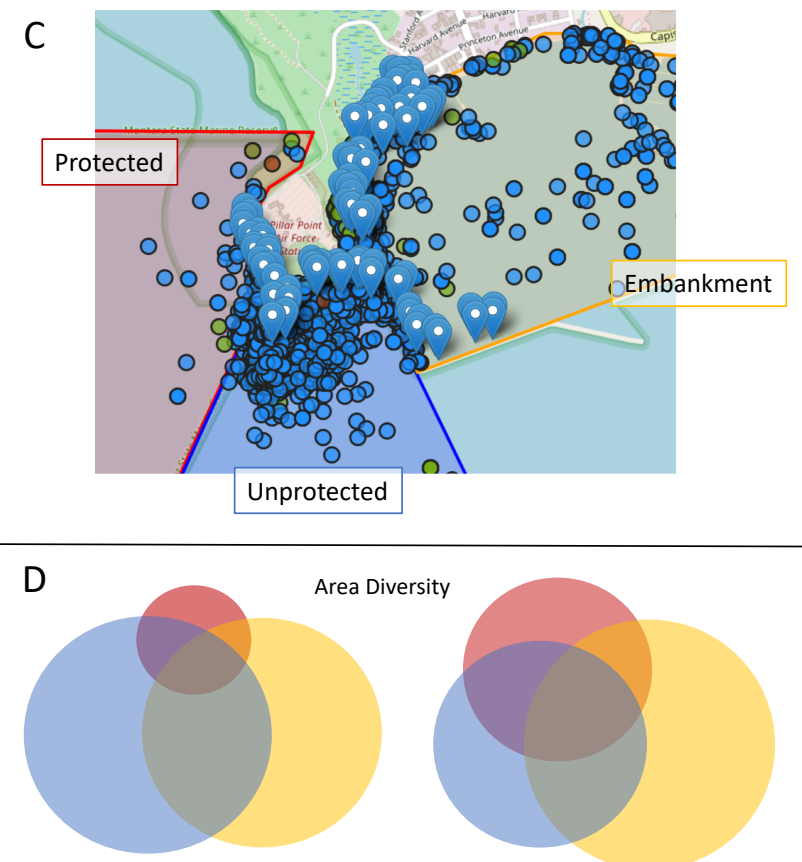

GBIF

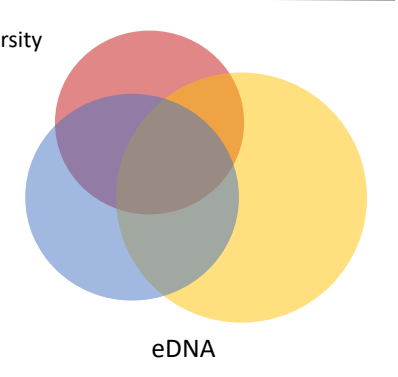

614

615

616

617

618

619

620

621

622

623

624

625

626 
627 Figure 4. Project vignettes. a. NHMLA program coordinator [NAME OBSCURED]

628 moves algae to uncover sediment for eDNA sampling by volunteers (inset). b. Left: the

629 coastal bioblitz sampling scheme that occurs in the same weekend. Right: volunteer

630 sampling the beach. c. Sampling the UC Merced Vernal Pool and Grassland Reserve.

631 Biologists introduce their research to volunteers (here, [NAME OBSCURED], professor

632 from CSULA, left, talks about fairy shrimp). d. Professors can be community scientists

633 too: here [NAME OBSCURED], professor from CSUMB, hikes at UC Fort Ord Natural

634 Reserve to collect for CALeDNA. e. [NAME OBSCURED] (left) samples along a lagoon.

635 Volunteers (right) help count organisms using traditional ecology methods. f.

636 Volunteer-submitted photos of paired burn samples from the Whittier Fire area. g.

637 [NAME OBSCURED] sampling in the Mojave desert. She is now the CALeDNA web

638 programmer. h. Left: Taxonomic richness is similar among the natural areas samples for

639 the desert project. Oak Glen is a non-desert sample representative of DNA found in

640 foothills that could wash into desert areas by runoff. Right: Presence of a taxon group

641 (y-axis) across desert samples (x-axis). Variation prompts questions about ecological

642 interactions among the stable members of the communities. 
bioRxiv preprint doi: https://doi.org/10.1101/503383; this version posted January 4 , 2019. The copyright holder for this preprint (which was not certified by peer review) is the author/funder, who has granted bioRxiv a license to display the preprint in perpetuity. It is made available under aCC-BY-NC-ND 4.0 International license.
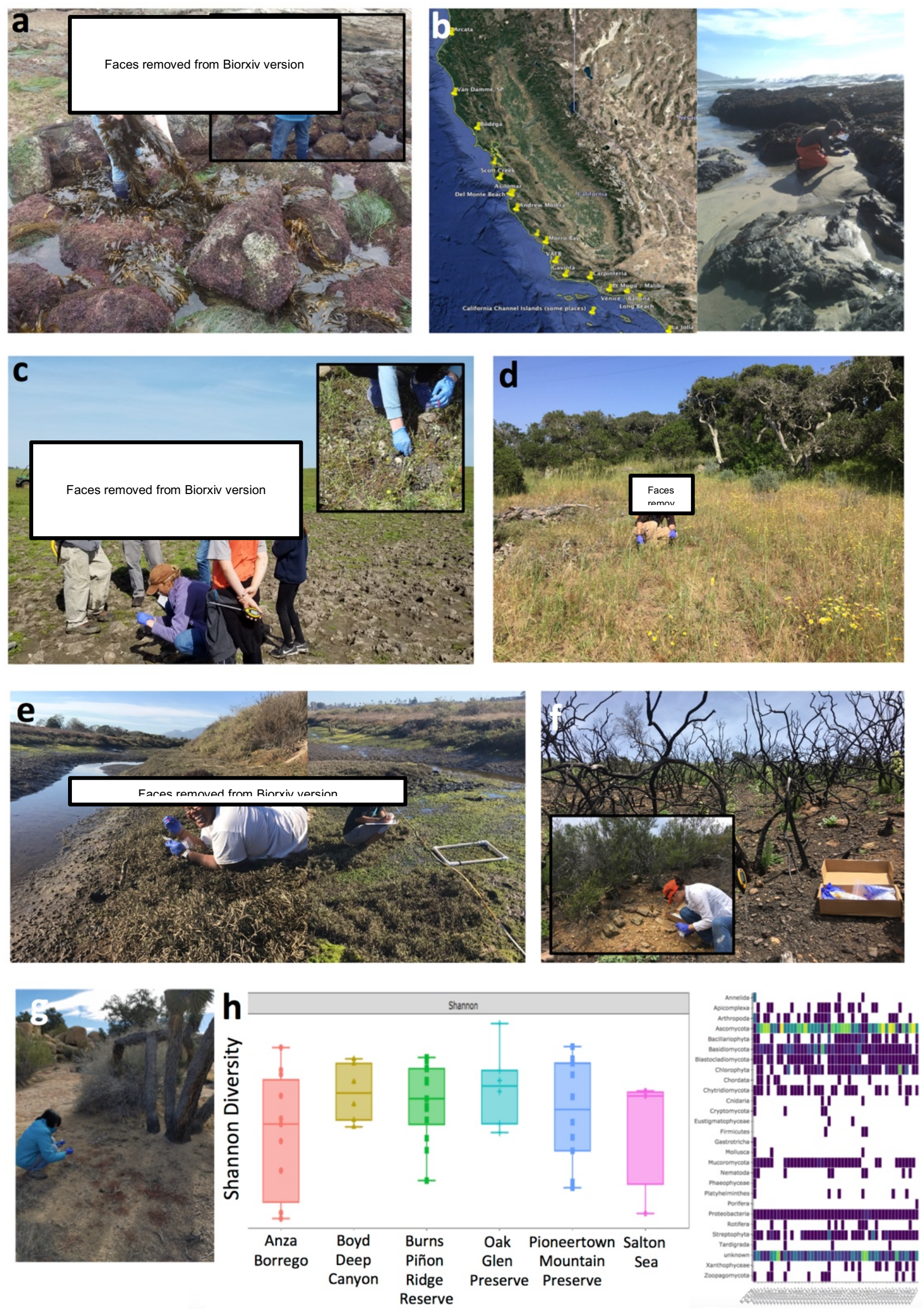
653 Figure 5. Taxonomic diversity plotted as a) unique taxon richness among phyla in

654 results using different primers in metabarcoding to amplify different regions of DNA.

$65516 S$ was chosen to amplify from bacteria and archaea. 18S was chosen to amplify the

656 broad diversity of eukaryotes. CO1 was chosen to amplify DNA from animals. FITS

657 (also called fungal ITS) was chosen to amplify all fungi. PITS (also called plant ITS2)

658 was chosen to amplify DNA from angiosperms. The specific primers and methods used

659 are on the CALeDNA website Methods for Researchers section:

660 www.ucedna.com/methods-for-researchers. The ten most commonly found phyla were

661 shown here. b. Non-metric multidimensional scaling plot (NMDS) showing beta

662 diversity is similar for scrub and woodland habitats (left cluster), and these are very

663 different from coastal samples (right). Each point represents one sample site, colored by

664 the minor habitat it belong to. Habitat definitions from

665 https://www.wildlife.ca.gov/Data/CWHR/Wildlife-Habitats.

666

667 Figure 5.

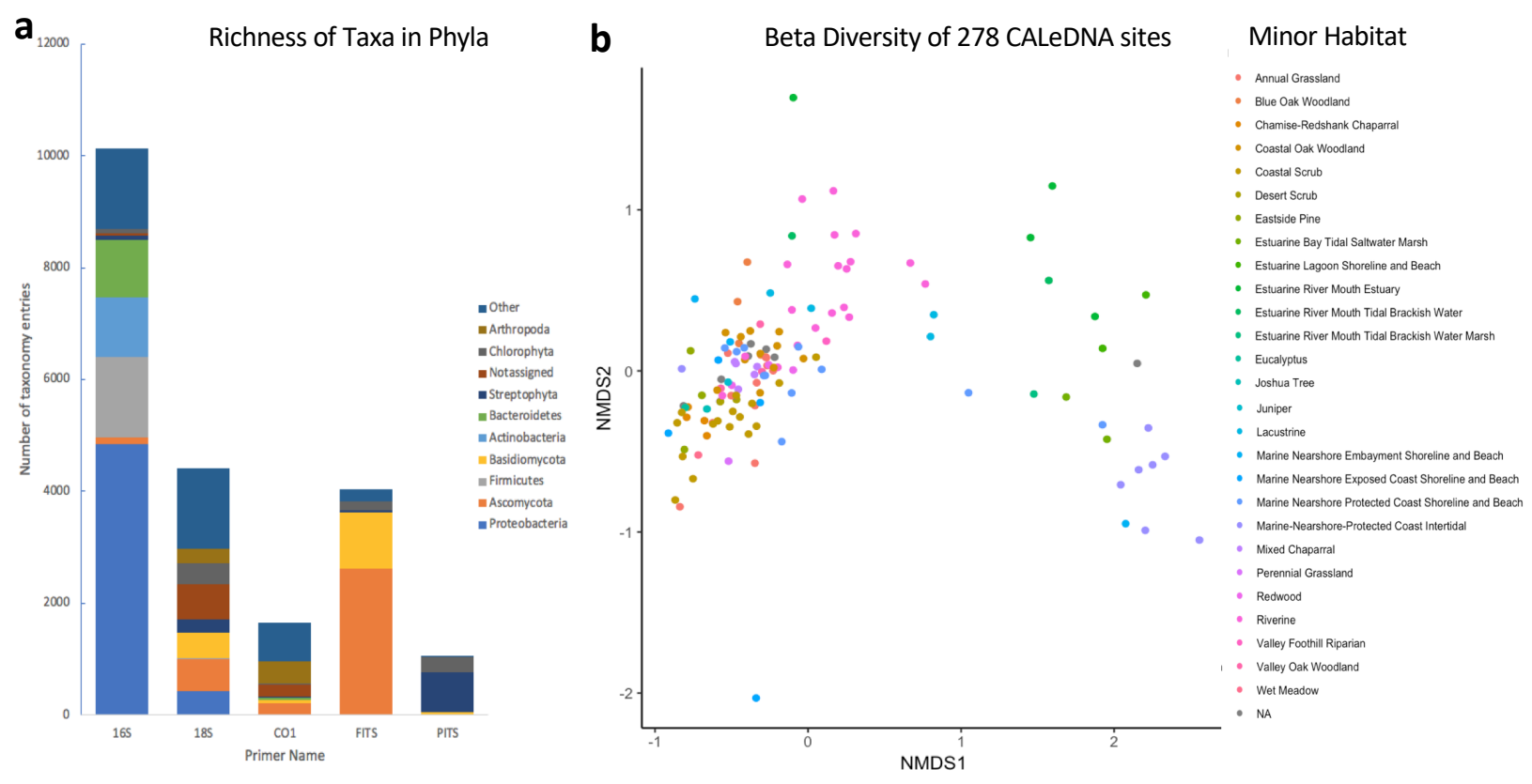


671 Figure 6. Left. The California's DNA: A field course sampling locations for Fall 2018.

672 Site number 1 is Carpinteria Salt Marsh Reserve, 2 is Stunt Ranch Reserve, 3 is the

673 Skirball area that burned in 2017, 4 is Franklin Canyon Park, 5 is the Los Angeles River

674 (Arroyo Seco), and 6 is the James San Jacinto Mountains reserve. The map was

675 generated in Google Earth Pro. Right. Participants at the CALeDNA Summer Research

676 Institute in Los Angeles. From left to right, [NAMES OBSCURED].

680 Figure 6.
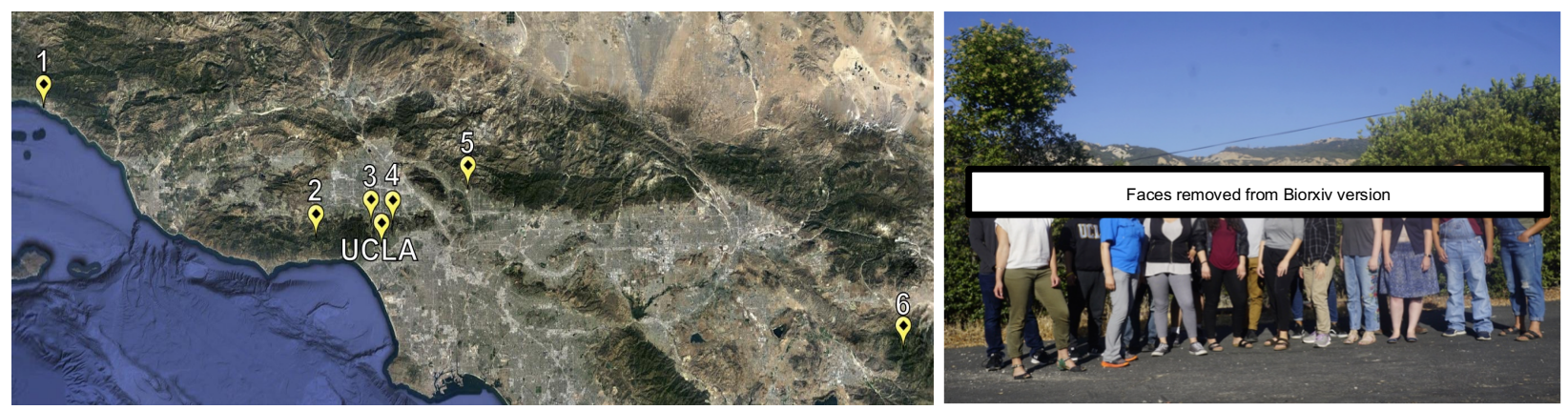

685 Note: Underlined words in the main text are intended for a sidebar glossary throughout 686 the paper.

688 Sidebar Glossary (will appear in the order they are introduced in the text): 
eDNA (environmental DNA): DNA from environmental samples such as soil, air,

690 surfaces, or water rather than directly from an organism. The DNA in the sample may

691 be shed from a living or dead organism such as from skin cells, or from an entire

692 organism that was collected as part of the sample, such as from a microbe. eDNA

693 degrades over time as it is exposed to the elements, and so where and how long it can

694 be detected depends on characteristics of the environment.

695 Bioblitz: hands-on, educational and fun community science activities such as bird or

696 wildflower surveys. They usually occur in a day and often contribute to biological

697 research, monitoring projects, or research resources (e.g., iNaturalist).

698 UC Natural Reserve System: A network of 39 (soon 40) natural reserves across

699 California that total 756,000 acres of land, and 50 miles of coastal shoreland (ucnrs.org).

700 The reserves function to save representatives of all of California's ecosystems for

701 research, education, and public service.

702 DNA barcodes: Short DNA sequences of a region that varies in sequence among species

703 and therefore can be used to match DNA to a species or strain. DNA barcodes are

704 usually sequenced from voucher specimens.

705 Metabarcoding: Sequencing a specific DNA barcode region of a genome from multiple

706 organisms within a single sample. The many resulting sequences are matched to known

707 DNA barcodes allowing variants to be assigned to identify species present.

708 Polymerase Chain Reaction (PCR): A technique used in molecular biology to make

709 many copies of a region of DNA to allow for sequencing. It is performed by adding a

710 mixture of enzymes, free nucleotides, buffers and primers to DNA, and then putting the

711 mixture through a series of specific heating and cooling incubations. Primers are short

712 sequences designed to flank the segment targeted for copying and sequencing. 
713 Voucher specimen: A whole organism or part thereof, such as a plant cutting for an

714 herbarium specimen, that is preserved for scientific use and used as a reference to

715 confirm identity.

716 NASA pre-HyspIRI flights: Since 2012, NASA has flown planes over parts of

717 California, with priority over UC natural reserves, to collect various kinds of remote

718 sensing data that describe the abiotic and biotic features of the local environment at

719 high resolution. These data inform the HyspIRI satellite design under plan to launch in

$720 \quad 2020$.

721 FAIR Data Principles: Principles of minimum standards for digital science information

722 distribution to benefit data providers and data consumers, both machine and human,

723 that were set in 2014 (FORCE11.org). Data should be Findable, $\underline{\text { Accessible, }}$

724 Interoperable, and Re-usable.

725 Alpha diversity: the mean species diversity or taxonomic richness in a location.

726 Beta diversity: a measure of diversity between areas, which helps describe diversity

727 turnover at a regional scale. Beta diversity accounts for the number of taxa common to

728 both areas and the number of unique taxa in each area. It describes the change in

729 community composition from location to location.

730 iNaturalist: A community platform for photographing, geotagging, and identifying

731 organisms. iNaturalist is a phone app maintained by the California Academy of

732 Sciences. To date, nearly 187,000 species have been observed in 15,000,000 observations

733 by $1.1 \mathrm{M}$ people.

734 Global Biodiversity Information Facility: A web-accessible database of all species

735 observations and collections. It houses information for $>1 \mathrm{~B}$ species occurrence records.

736 DNA data have only just begun to be included as an 'observation' of a species (UNITE;

737 GBIF 2018). 
bioRxiv preprint doi: https://doi.org/101101/503383; this version posted January 4, 2019. The copyright holder for this preprint (which was not certified by peer review) is the author/funder, who has granted bioRxiv a license to display the preprint in perpetuity. It is made available under aCC-BY-NC-ND 4.0 International license.

738 Flipped Learning Courses: Courses where content is learned via media at home and

739 classroom time is used to carry out exercises that apply content.

740

741 